\title{
Liposomes: from biophysics to the design of peptide vaccines
}

\section{F. Frézard}

\section{Correspondence}

F. Frézard

Departamento de Fisiologia e

Biofísica, ICB, UFM G

Av. Antônio Carlos, 6627

30161-901 Belo Horizonte, MG

Brasil

Fax: +55-31-499-2924

E-mail: frezard@ mono.icb.ufmg.br

Presented at the International

Symposium "The Third Revolution on Vaccines: DNA Vaccines",

Belo Horizonte, MG, Brasil,

November 3-7, 1997.

Research supported by $\mathrm{CNPq}$ (No. 521010/97), FAPEMIG (No. CBS1721/95) and PRONEX (No. 3075).

Received November 6, 1998 Accepted November 27, 1998
Departamento de Fisiologia e Biofísica, Instituto de Ciências Biológicas, Universidade Federal de Minas Gerais, Belo Horizonte, MG, Brasil

\section{Abstract}

Liposomes (lipid-based vesicles) have been widely studied as drug delivery systems due to their relative safety, their structural versatility concerning size, composition and bilayer fluidity, and their ability to incorporate almost any molecule regardless of its structure. Liposomes are successful in inducing potent in vivo immunity to incorporated antigens and are now being employed in numerous immunization procedures. This is a brief overview of the structural, biophysical

Key words

- Liposome

- Immunoadjuvant

- Immunostimulant

- Vaccine

- Encapsulation and pharmacological properties of liposomes and of the current strategies in the design of liposomes as vaccine delivery systems.

\section{Introduction}

Almost 75 years ago, Paul Ehrlich established the concept of the "magic bullet" envisioning a drug delivery mechanism that would target drugs directly to diseased cells. It was not long after liposomes were first constructed by A.D. Bangham in the early 1960s, that it was demonstrated that a wide variety of molecules could be encapsulated within the aqueous spaces of liposomes or inserted into their membranes. It was generally assumed at that time that since liposomes were primarily made of natural phosphatidyl choline, liposome-encapsulated antigen would avoid the mononuclear phagocyte system (MPS) clearance and would not be recognized as a particulate antigen. However, it was rapidly realized that eventually liposomes would be deposited at high concentrations in the MPS organs, particularly in fixed macrophages $(1,2)$, regardless of the composition or size of the vesicles. Later, the capture of liposomes by macrophages was recognized as the main mechanism by which liposomes potentiate immune re- sponses to entrapped antigens. The pioneer work of Allison and Gregoriadis (3), that demonstrated the immunoadjuvant properties of liposomes, was followed by a multitude of related animal immunization studies (4-7). Finally, liposomes as adjuvants have come of age, with the first liposome-based vaccine (against hepatitis $A$ ) having been licensed for use in humans (8).

\section{Structure and biophysical properties of liposomes}

Liposomes are artificial vesicles composed of concentric lipid bilayers which are separated by water compartments. The typical characteristic of bilayer-forming lipids is their amphiphilic nature: a polar headgroup covalently attached to one or two hydrophobic hydrocarbon tails. Structures of the most commonly used bilayer-forming lipids are illustrated in Table 1. When these lipids are exposed to an aqueous environment, interactions between themselves (hydrophilic interactions between polar headgroups and Van der Waals' interactions between hydrocar- 
bon chains) and with water (hydrophilic interactions, hydrophobic effect) lead to spontaneous formation of closed bilayers.

Liposomes can differ in size: they can range from the smallest vesicle obtainable on theoretical grounds (diameter $\sim 20 \mathrm{~nm}$ ) to liposomes which are visible under the light microscope, with a diameter of $1 \mu \mathrm{m}$ or greater, equal to the dimensions of living cells. They can also differ in terms of lipid composition and structural organization, corresponding to uni-, oligo- or multi-lamellar vesicles. Liposomes are built in such a way that the solute can be encapsulated in the aqueous compartment (polar solutes) or embedded in the lipid bilayers (lipophilic or amphiphilic solutes).

The properties of liposomes and their subsequent applicability depend on the physical and physico-chemical characteristics of the liposomal membrane. Usually, a zwitterionic or non-ionic lipid is used as the basic lipid for the preparation of liposomes (Table 1). The net surface charge of liposomes can be modified by the incorporation of positively charged lipids, such as stearylamine, or negatively charged lipids, such as dicetylphosphate, phosphatidyl glycerol (Table 1) or phosphatidyl serine. Bilayer elasticity is related to elastic properties such as tensile strength, compressibility and bending. This property has been used to understand the response of bilayers to mechanical stress and to manufacture liposomes. The fluidity of the liposomal bilayer, when it is made from a single lipid, depends on the lipid phase transition temperature (Tc) and its relative position compared to ambient temperature. When ambient temperature is increased and reaches Tc, the membrane passes from a "solid" gel phase, where the lipid hydrocarbon chains are in an ordered state, to a "fluid" liquid-crystal phase, a disordered state, where molecules have more freedom of movement (2). Hence, depending on lipid Tc (Table 1), different membranes composed of distinct lipids can exhibit different fluidity levels at the same temperature. The bilayer permeability is a measure of the flux or rate at which a solute works its way from an aqueous compartment, through a bilayer, and out into the aqueous compartment on the other side. It depends on the membrane fluidity and on the nature of the solute. Membrane permeability is highest at the phase transition temperature, and is lower in the gel phase than in the fluid phase. A general sequence of hydrophilic solute permeability is: water $>$ small non-electrolytes $>$ anions $>$ cations $\cong$ large non-electrolytes $>$ large polyelectrolytes (2).

The fusion properties of liposomal membranes have recently gained special attention with the discovery of lipid compositions that allow for the delivery of macromolecules into the cytoplasm of cells $(9,10)$. Membrane fusion is promoted by bilayer dehydration (11), by acidification when the membrane contains $\mathrm{pH}$-sensitive lipids, such as DOPE (12), and by the presence of cationic lipids or of anionic lipids with divalent cations.

\section{Methods for liposome preparation and solute encapsulation}

Since the early 1970 s, many hundreds of drugs, including antitumor and antimicrobial agents, chelating agents, peptide hormones, enzymes, other proteins, vaccines and genetic materials, have been incorporated into the aqueous or lipid phase of liposomes of various sizes, compositions and other characteristics by an ever increasing number of technologies (13). The successful evolution of liposomes from experimental tools to industrially manufactured products for clinical and veterinary use depends on efficient drug entrapment using simple, reproducible and inert methods. In the case of an antigenic protein or peptide, such association may be obtained by covalent attachment of the antigen to the liposome surface (14), or take the form of entrapment into the 
aqueous phase of vesicles, adsorption onto their surface, or partition into the bilayers (2). Among available methods for peptide or protein encapsulation, three are outstanding for higher encapsulating efficiency. The reverse-phase evaporation technique, the first to use 'water-in-oil' emulsions (15), encapsulates up to $50 \%$ of solute. Preparation of reverse-phase evaporation vesicles (REV) consists of a rapid injection of aqueous solution into an organic solvent which contains the lipids dissolved. Thus, following the formation of water droplets ('water-in-oil' emulsion) by bath sonication of the two-phase mixture, the emulsion is dried down to a semi-solid gel in a rotary evaporator. The next step is to subject the gel to vigorous mechanical shaking to induce a phase change from a water-in-oil emulsion to a vesicle suspension. In these circumstances, some water droplets collapse, and these droplets attach to adjacent, intact vesicles to form the outer leaflet of the bilayer of a large unilamellar liposome (diameter in the range of 0.1 to $1 \mu \mathrm{m})$.

Another method (16) that produces dehydration-rehydration vesicles (DRV) is both simple and easy to scale up, and usually gives high yields of solute entrapment (up to $80 \%)$ (16-20). Another advantage of the DRV method compared to the REV method is that it does not expose the solute to potentially denaturating organic solvents and/or to sonication. Preparation of DRV consists of mixing an aqueous solution of the solute with a suspension of 'empty' (water-containing) liposomes and freeze-drying the resulting mixture. The intimate contact of flattened liposomal membrane structures and solute molecules in a dry environment and the fusion of

Table 1 - Examples of bilayer-forming lipids used to prepare liposomal vaccines.

Tc, Transition temperature.

\begin{tabular}{lcc}
\hline $\begin{array}{l}\text { Lipid family } \\
\text { Structural formula }\end{array}$ & $\begin{array}{c}\text { Hydrophobic chains (R) } \\
\text { (name) }\end{array}$ & Abbreviation of lipid name (Tc) \\
\hline
\end{tabular}

Phosphatidyl choline

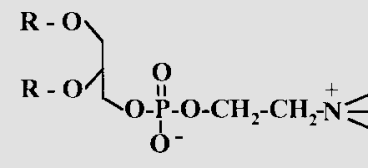

Phosphatidyl ethanolamine

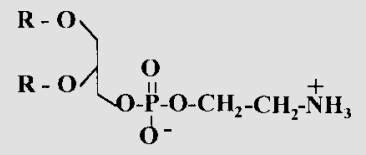

Phosphatidyl glycerol

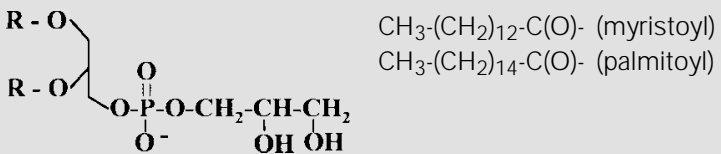

Alkyl polyoxyethylene ether (example of non-ionic surfactant)

R - $\left(\mathrm{O}-\mathrm{CH}_{2}-\mathrm{CH}_{2}\right)_{6}-\mathrm{CH}_{2}-\mathrm{CH}_{2} \mathrm{OH} \quad \mathrm{CH}_{3}-\left(\mathrm{CH}_{2}\right)_{15}-$ (cetyl)
DOPC $\left(<0^{\circ} \mathrm{C}\right)$

DMPC $\left(+23^{\circ} \mathrm{C}\right)$

DPPC $\left(+42^{\circ} \mathrm{C}\right)$

DSPC $\left(+55^{\circ} \mathrm{C}\right)$

$$
\mathrm{CH}_{3}-\left(\mathrm{CH}_{2}\right)_{7}-\mathrm{CH}=\mathrm{CH}-\left(\mathrm{CH}_{2}\right)_{7}-\mathrm{C}(\mathrm{O}) \text { - (oleyl) DOPE }\left(<0^{\circ} \mathrm{C}\right)
$$

DMPG $\left(+13^{\circ} \mathrm{C}\right)$

DPPG $\left(+35^{\circ} \mathrm{C}\right)$ 
membranes caused by dehydration facilitates the incorporation of solute during the controlled rehydration steps. Separation of solute-containing DRV from unentrapped solute can be carried out easily if needed (by centrifugation, for example). Vesicles formed by the dehydration-rehydration technique are multilamellar with heterogeneous sizes (diameters varying from 0.1 to $2.0 \mu \mathrm{m}$ ).

The third method requires the use of detergent (21). Lipids are first solubilized with an aqueous solution of the detergent that also contains the protein(s) to be encapsulated. The detergent should have a high critical micelle concentration (CMC) so that it is easily removed, for instance by dialysis. During detergent removal, relatively small liposomes (mean diameter in the range of 0.08 to $0.2 \mu \mathrm{m}$ ) with a narrow size distribution will be produced. This latter method was found to be particularly suitable for the reconstitution of membrane proteins in liposomes.

\section{Stability of liposome preparations}

The most important use of liposomes results from their ability to retain solutes for long periods of time. Other important aspects of the stability of liposomes refer to the maintenance of their size distribution and to the chemical stability of their content. Liposome stability has to be considered under storage and physiological conditions. Under physiological conditions, solute leakage depends on membrane permeability and also on the interaction with components of biological fluids. In serum, the lipid molecule can be transferred from the liposomal membrane to plasma high density lipoprotein. This is particularly true in the case of "fluid" liposomes, such as those made from dioleyl-phosphatidylcholine (DOPC), which disintegrate and release their contents within few minutes after their intravenous administration. Following the substitution of DOPC by high phase transition temperature lipids such as distearoyl-phosphatidylcholine (DSPC), the bilayer becomes "rigid" at $37^{\circ} \mathrm{C}$, and consequently resistant to lipoprotein attack. Membrane fluidity can also be controlled quite accurately by supplementing the lipid bilayer with cholesterol, a mechanism that results in enhanced membrane stability $(1,2)$, by mixing two or more lipids, or by manipulating the hydrophobic/ lipophobic character of the bilayers, for example with the use of fluorinated lipids (2224). The rate of solute leakage also depends on the lamellarity of liposomes, multilamellar vesicles being more stable than unilamellar ones (16). When given by the oral route, liposomes have to survive the "detergent effect" of bile salts and phospholipase activity. Only the most "rigid" liposomes were found to resist these extreme conditions (25).

Liposomes, once formed, must be stored in a form that confers long-term stability to the liposome preparation (encapsulation, size and chemical stability) and their contents. For instance, the premature release of solute from stored liposomes makes encapsulation ineffectual and may increase the toxicity of the formulation when it is used. Liposomes may be stored as freeze-dried powders, however, care must be taken to avoid the dehydration-induced phase transition and membrane fusion. Indeed, membrane fusion results in the increase of liposome size and in the partial release of entrapped solute. The cryoprotective effect of sugars on liposome integrity has been extensively studied $(11,26,27)$. Sugars were found to preserve the reactivity and immunogenicity of functional groups entrapped (14). Studies on freeze-dried liposomes have indicated trehalose as the most effective sugar to preserve liposome integrity. This effect has been attributed to the inhibition of membrane fusion and to the decrease of the Tc of dry lipids, thus avoiding membrane phase transition. 


\section{In vivo fate of liposomes}

Most of our knowledge concerning the behavior of liposomes in vivo has been obtained using intravenous injections. Liposomes given intravenously normally interact with at least two distinct groups of plasma proteins (28): i) the plasma high density lipoproteins and ii) the so-called opsonins which, by adsorbing onto the surface of vesicles, mediate their endocytosis by MPS macrophages. The rate of liposome clearance from the blood circulation will therefore depend on the ability of opsonins to bind to the liposome surface and can be manipulated through the appropriate selection of liposome characteristics $(1,4)$. For instance, "fluid" vesicles are removed more rapidly from blood circulation than "rigid" ones. It was suggested that opsonins did not adsorb as avidly on vesicles with rigid bilayers. Clearance from the blood stream is also influenced by surface charge (29) and by vesicle size. The longest half-life is obtained when liposomes are relatively small (diameter $<0.05 \mu \mathrm{m}$ ) and carry no net surface charge. However, regardless of the time of liposome persistence within the vascular system, much of an injected dose is taken up by MPS macrophages, ending up in the lysosomal apparatus. There, liposomes are disrupted within the lysosomes, the solute is released locally and, depending on its nature, can either remain in the lysosome, be transferred into the cytoplasm, or diffuse out from cells.

The behavior of preparations given by alternative parenteral routes, such as the intraperitoneal, subcutaneous or intramuscular route, is influenced by the distribution of liposome size and their lipid composition and will also depend on the route of injection (30). A proportion of liposomes enter the lymphatic system and, eventually, the blood circulation where they behave as if given intravenously. However, whereas liver, spleen and bone marrow take up nearly all liposomes given by the intravenous route, they will account for a much smaller proportion if the subcutaneous or intramuscular route is used. The remainder (up to $80 \%$ of liposomes) is retained at the site of injection and attacked by infiltrating macrophages or intercepted in the local lymph nodes.

Prolonged survival of liposomes in the circulation is required when these are designed to act on non-MPS tissues, within the vascular system, extravascularly through leaky capillaries or as circulating slow drug release systems. Recent works have shown that coating the liposome surface with polyethyleneglycol and other hydrophilic polymers (31), or chemically modifying the hydrophobic part of phospholipids $(23,32)$, substantially prolongs the half-life of liposomes in the blood. Furthermore, these liposomes have a propensity to accumulate in implanted tumors at levels compared to other non-MPS tissues.

\section{Liposomes as immunological adjuvants}

New-generation vaccines that are based on recombinantly made subunit and synthetic-peptide antigens are usually nonimmunogenic, and the need for immunopotentiation is well recognized. Although many structurally unrelated agents (immunological adjuvants) are capable of inducing immune responses to vaccine antigens, most of them are toxic. Surprisingly, for about 70 years the only immunological adjuvant licensed for use in humans was, until recently, aluminum salts (alum). However, they are far from ideal: they are not always effective, induce humoral immunity (HI) but not cellmediated immunity (CMI), and cannot be lyophilized. Twenty-five years after the discovery of the immunological adjuvant properties of liposomes, they appear now as a major candidate adjuvant, with a liposome- 
based vaccine (against hepatitis A) being licensed for use in humans (8). Vaccines based on novasomes $(8,13,33)$ (nonphospholipid liposomes formed from single-chain amphiphiles, with or without other lipids) have been licensed for the immunization of fowl against Newcastle disease virus and avian rheovirus. Other liposomal or novasome-based vaccines against bacterial and viral infections are under development. Liposomes offer a number of advantages as carriers of vaccines (4) in that they are biodegradable and nontoxic, can elicit both HI and CMI (7), and can be prepared entirely synthetically. Furthermore, they are highly versatile in their structural characteristics, which allow for the precise manipulation of their immunoadjuvant properties. A number of structural variables of liposomes can influence adjuvanticity $(4,6)$ : the lipid to antigen mass ratio, bilayer fluidity, vesicle size, surface charge (34), and the mode of antigen association with the vesicles. Manipulation of these variables usually induces variation in the immune response level of a maximal factor of three (7). To boost the response further, different immunostimulants have been tested. Among them are avridine, muramyl-dipeptide (MDP) and MDP-lipid conjugates, nonionic block polymer surfactants, aluminum salts, IL-2, IL-6 and lipid A. It is noteworthy that the impact of the incorporation of these immunostimulants into liposomes was not only an increase of their immunological action, but also a reduction of their toxic side effects (5). Because of the complexity of the interrelationship between the above-mentioned parameters, it is difficult, at the present time, to establish general rules to maximize the immunogenicity of liposomal vaccines or to direct immune responses. However, the insights into the manipulation of immune responses by the choice of liposome characteristics are growing.

A lot of work has been done to study the interaction of liposomes with cells of the immune systems and to elucidate the mechanism of induction of immune reactions (4). It is generally accepted that a physical association between liposomes and antigen (as

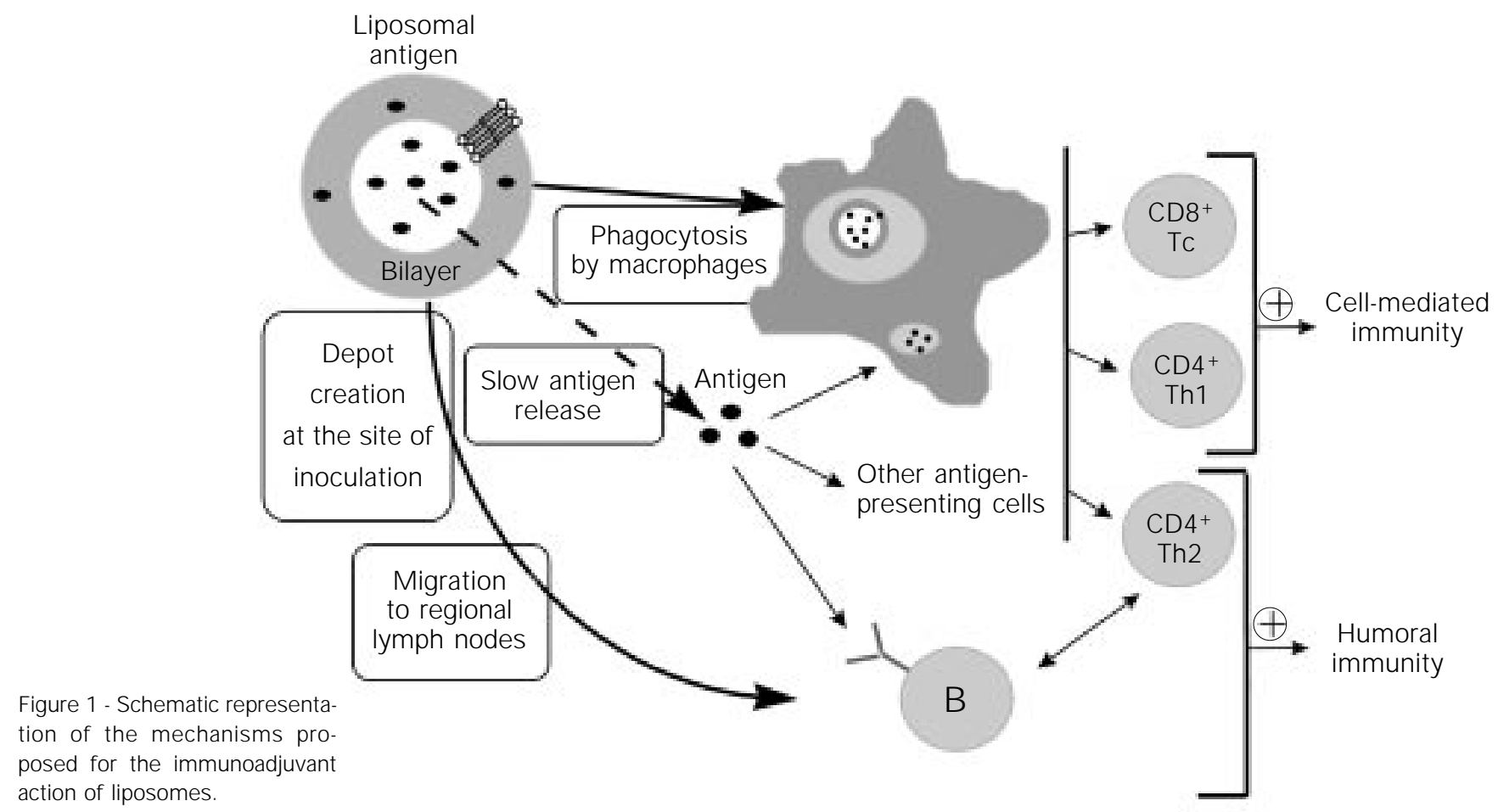


opposed to their simple mixing) is a prerequisite for adjuvanticity to occur. The current presumed mode of action of liposomes is illustrated in Figure 1. The enhancement of $\mathrm{HI}$ to antigens by means of their incorporation into liposomes can be attributed to the generation of a depot at the site of injection which prolongs the release and interaction of free or liposome-associated antigens with antigen-presenting cells (APC). Among APC, macrophages were shown to play a major role, because of their unique ability to phagocytize liposomes. These cells are also expected to invade the depot area in response to local inflammation. Finally, a fraction of liposomal antigen will migrate to areas in the regional lymph nodes containing $\mathrm{T}$ cells. This fate of liposomes, in addition to their ability to efficiently present antigens in a hydrophobic environment, may be responsible for the stimulation of CMI (6). It is noteworthy that liposomes, in addition to promoting immunity to antigens injected through a variety of parenteral routes, also increase $\operatorname{IgA}$ immunity to antigens given orally, probably because of vesicle interaction with the gut lymphoid tissue (25). Recently, more detailed information has become available on the fate of liposomes after uptake by cells and the subsequent induction of cytotoxic T lymphocyte responses. Liposomes made from dioleyl-phosphatidylethanolamine (DOPE) were found to be $\mathrm{pH}$ sensitive $(9,12)$. Upon protonation of DOPE at a $\mathrm{pH}$ below 6.5, DOPE-containing bilayers undergo a transition from the bilayer phase to the hexagonal phase. Under these conditions, liposomes are destabilized and become fusogenic. Using $\mathrm{pH}$-sensitive and $\mathrm{pH}-$ insensitive ("classical") liposomes, it has been possible to selectively trigger class I- or class II-restricted immune responses, at least in vitro (35). Liposomes were taken up by endocytosis. Acid-sensitive vesicles were destabilized in the acidic endosome, fused with the endosomal membrane and released liposomal antigen into the cytoplasm. They were then transported to the endoplasmic reticulum where they combined with class I molecules. On the other hand, acid-resistant vesicles were delivered to the lysosome, where they were degraded and transported to the late endosome. There, they combined with class II molecules. In vivo, no difference in immune response between the two types of liposomes could be discerned. Both $\mathrm{pH}$-sensitive and -insensitive liposomes were able to induce class I-restricted immune responses in vivo. Therefore, under the chosen conditions, $\mathrm{pH}$ sensitivity for liposomes does not seem to be absolutely necessary.

\section{Concluding remarks}

Liposomal adjuvanticity appears to depend on several of the structural characteristics of the system which are known to determine its fate in vivo and, thus, the mode of interaction with APC. Adjuvanticity is further improved by the presence of other adjuvants. To date, the story of liposomes as vaccine-delivery systems appears to be a success. It has come about as a result of the accumulated knowledge of their interaction with in vivo systems, which has permitted the rational design of vesicle constructs, and through the sophisticated advances in liposome technology. Clearly, this is only the beginning, and there are great challenges. The elucidation of the mechanism of liposomal immunoadjuvant activity at the tissue, cellular and subcellular levels is a formidable task, but could help to establish the roles of the spatial arrangement of antigen within the structure of vesicles, and of their structural characteristics. This would in turn contribute to the design of constructs for specific tasks such as the preferential elicitation of humoral or cell-mediated immunity, the selective increase of protective antibody isotypes and the preferential induction of class-I or class II-restricted immune responses. Some recent new developments are also promising. For instance, the incorpora- 
tion of antigen-carrying liposomes into microspheres, that permits single dose immunization with built-in booster effects, is expected to increase the immunogenicity of the preparation (36). Finally, an exciting new field in vaccinology is the use of nucleic acids as (pre-)antigens (37).

\section{Acknowledgments}

I thank Dr. Thais V. de Freitas for her help and suggestions about the preparation of the manuscript.

\section{References}

1. Ostro MJ (1987). Introduction. In: Ostro MJ (Editor), Liposomes: from Biophysics to Therapeutics. Marcel Dekker, Inc., New York and Basel.

2. New RRC (1990). Introduction. In: New RRC (Editor), Liposomes - a Practical Approach. IRL Press, Oxford, New York, Tokyo.

3. Allison AC \& Gregoriadis G (1974). Liposomes as immunological adjuvants. $\mathrm{Na}$ ture, 252: 252.

4. Gregoriadis G (1990). Immunological adjuvants: a role for liposomes. Immunology Today, 11: 89-97.

5. Alving CR (1991). Liposomes as carriers of antigens and adjuvants. J oumal of Immunological Methods, 140: 1-13.

6. Gregoriadis G (1993). Liposome as immunological adjuvants for peptide and protein antigens. In: Gregoriadis G, Florence AT \& Patel AM (Editors), Liposomes in Drug Delivery. Harwood Academic Publishers, Switzerland.

7. Kersten GFA \& Crommelin DJ A (1995). Liposomes and ISCOMS as vaccine formulations. Biochimica et Biophysica Acta, 1241: 117-138.

8. Gregoriadis G (1995). Engineering liposomes for drug delivery: progress and problems. Trends in Biotechnology, 13: 527-537.

9. Connor J , Yatvin M \& Huang L (1984). PHsensitive liposomes: acid induced fusion. Proceedings of the National Academy of Sciences, USA, 81: 1715-1720.

10. Legendre J -Y \& Szoka J r FC (1995). Liposomes for gene therapy. In: Puisieux $F$, Couvreur P, Delattre J \& Devissaguet J P (Editors), Liposomes, New Systems and New Trends in Their Applications. Editions de Santé, France.

11. Crowe JH, Hoekstra FA \& Crowe LM (1992). Anhydrobiosis. Annual Review of Physiology, 54: 579-599.

12. Allen TM, Hong $K \&$ Papahadjopoulos $D$ (1990). Membrane contact fusion and hexagonal (HII) transitions in phosphatidyl ethanolamine liposomes. Biochemistry, 29: 2976-2985.

13. Gregoriadis G (1993). Liposome Technology. 2nd edn. CRC Press Inc., Boca Raton, $\mathrm{FL}$.

14. Friede $M$, van Regenmortel $M H V$ \& Schuber F (1993). Lyophilized liposomes and shelf items for the preparation of immunogenic liposome-peptide conjugates. Analytical Biochemistry, 211: 117-122.

15. Szoka F \& Papahadjopoulos D (1978). Procedure for preparation of liposomes with large internal aqueous space and high capture by reverse-phase evaporation. Proceedings of the National Academy of Sciences, USA, 75: 4194-4198.

16. Kirby $C \& \&$ Gregoriadis $G$ (1984). Dehydration-rehydration vesicles: a simple method for high yield drug entrapment in liposomes. Biotechnology, 2: 979-984.

17. Shew RL \& Deamer DW (1985). A novel method for encapsulation of macromolecules in liposomes. Biochimica et Biophysica Acta, 816: 1-8.

18. Tan L, Loyter A \& Gregoriadis G (1989). Incorporation of reconstituted influenza virus envelopes into liposomes: studies of the immune response in mice. Biochemical Society Transactions, 17: 129130.

19. Freitas TV \& Frézard F (1997). Encapsulation of native crotoxin in liposomes: a safe approach for the production of antivenom and vaccination against Crotalus durissus temificus venom. Toxicon, 35: 91-100.

20. Frézard $F$, Toledo VPCP, Elias RM, Tavares CAP, Da Costa CA, Genaro O \& Mayrink W (1995). Vaccination of C57BL/ 10 mice against cutaneous leishmaniasis by liposome-encapsulated antigens. Memórias do Instituto Oswaldo Cruz, 90 (Suppl I): 194 (Abstract IM-091).

21. Brunner J, Skrabal P \& Hauser H (1976). Single bilayer vesicles prepared without sonication. Physico-chemical properties.
Biochimica et Biophysica Acta, 455: 322331.

22. Frézard $F$, Santaella $C$, Montisci MJ Vierling P \& Riess J G (1994). Fluorinated phospholipid-based liposomes: $\mathrm{H}^{+} / \mathrm{Na}^{+}$ permeability, active doxorubicin encapsulation and stability in human serum. Biochimica et Biophysica Acta, 1194: 61-68.

23. Riess J G, Frézard F, Greiner J , Kraft MP, Santaella C, Vierling P \& Zarif L (1995). Membranes, vesicles and other supramolecular systems made from fluorinated amphiphiles. In: Barenholz Y \& Lasic D (Editors), Handbook of Non-Medical Applications of Liposomes. CRC Press Inc., Boca Raton, FL.

24. Frézard $F$, Santaella $C$, Vierling $P \&$ Riess J G (1994). Permeability and stability in buffer and in human serum of fluorinated phospholipid-based liposomes. Biochimica et Biophysica Acta, 1192: 61-70.

25. Fattal E, Ramaldes GA \& Ollivon M (1995) Oral delivery of vaccines by means of liposomes. In: Puisieux F, Couvreur P, Delattre J \& Devissaguet J P (Editors), Liposomes, New Systems and New Trends in Their Applications. Editions de Santé, France.

26. Crowe LM, Crowe JH, Rodolph A, Womersley C \& Appel L (1985). Preservation of freeze-dried liposomes by trehalose. Archives of Biochemistry and Biophysics, 242: 240-247.

27. Crowe LM \& Crowe JH (1995). Freezedried liposomes. In: Puisieux F, Couvreur P, Delattre J \& Devissaguet J P (Editors), Liposomes, New Systems and New Trends in Their Applications. Editions de Santé, France.

28. Devine DV \& Marjan J MJ (1997). The role of immuno-proteins in the survival of liposomes in the circulation. Critical Reviews in Therapeutic Drug Carrier Systems, 14: 105-131.

29. Liu F \& Liu D (1996). Serum independent liposome uptake by mouse liver. Biochimi- 
ca et Biophysica Acta, 1278: 5-11.

30. Oussoren C, Zuidema J , Crommelin DJ \& Storm G (1997). Lymphatic uptake and biodistribution of liposomes after subcutaneous injection. II. Influence of liposomal size, lipid composition and lipid dose. Biochimica et Biophysica Acta, 1328: 261272.

31. Lasic DD \& Papahadjopoulos D (1995). Liposomes revisited. Science, 267: 12751276.

32. Santaella $C$, Frézard $F$, Vierling $P \&$ Riess J G (1993). Extended in vivo blood circulation time of fluorinated liposomes. Federation of European Biochemical Socie- ties Letters, 336: 481-484.

33. Gupta RK, Varanelli CL, Griffin $P$, Wallach DF \& Sibber GR (1997). Adjuvant properties of non-phospholipid liposomes (Novasomes) in experimental animals for human vaccine antigens. Vaccine, 14: 219-225.

34. Nakanishi T, Kunisawa J, Hayashi A, Tsutsumi Y, Kubo K, Nakagawa S, Fujiwara H, Hamaoka T \& Mayumi T (1997). Positively charged liposomes function as an efficient immunoadjuvant in inducing immune responses to soluble proteins. Biochemical and Biophysical Research Communications, 240: 793-797.
35. Reddy R, Zhou F, Nair S, Huang L \& Rouse BT (1992). In vivo cytotoxic T lymphocytes induction with soluble proteins administered in liposomes. J ournal of Immunology, 148: 1585-1589.

36. Cohen S \& Langer R (1995). Long-term protein delivery from microencapsulated liposome systems. In: Puisieux F, Couvreur P, Delattre J \& Devissaguet J P (Editors), Liposomes, New Systems and New Trends in Their Applications. Editions de Santé, France.

37. Gregoriadis G (1998). Genetic vaccines: strategies for optimization. Pharmaceutical Research, 15: 661-670. 